\title{
Low serum sphingolipids in children with attention deficit-hyperactivity disorder
}

\author{
Marcela P. Henríquez-Henríquez ${ }^{1,2}$, Sandra Solari ${ }^{2}$, Teresa Quiroga ${ }^{2}$, Benjamin I. Kim ${ }^{3}$, \\ Richard J. Deckelbaum ${ }^{1}$ and Tilla S. Worgall ${ }^{3 *}$ \\ ${ }^{1}$ Department of Pediatrics, Institute of Human Nutrition, College of Physicians and Surgeons, Columbia University, New York, \\ NY, USA, ${ }^{2}$ Department of Clinical Laboratories, School of Medicine, Pontificia Universidad Católica de Chile, Santiago, Chile, \\ ${ }^{3}$ Department of Pathology and Cell Biology, Columbia University, New York, NY, USA
}

\section{OPEN ACCESS}

Edited by:

John Vijay Sagar Kommu National Institute of Mental Health and Neurosciences, India

Reviewed by:

Karen M. Smith,

University of Louisiana at Lafayette,

USA

José A. Alda,

Hospital Sant Joan

de Deu. Barcelona, Spain

Suhash Chakraborty,

Hindustan Aeronautics Limited

Hospital, India

*Correspondence: Tilla S. Worgall, Department of Pathology and Cell Biology, Columbia University, Black

Building 457, 430 W 168 St.,

New York, NY 10032, USA

tpw7@columbia.edu

Specialty section:

This article was submitted to Child and Neurodevelopmental

Psychiatry,

a section of the journal

Frontiers in Neuroscience

Received: 29 April 2015 Accepted: 07 August 2015

Published: 25 August 2015

Citation:

Henríquez-Henríquez MP, Solari S, Quiroga T, Kim BI, Deckelbaum RJ and Worgall TS (2015) Low serum sphingolipids in children with attention deficit-hyperactivity disorder

Front. Neurosci. 9:300

doi: 10.3389/fnins.2015.00300
Background: Attention deficit-hyperactivity disorder (ADHD) is the most prevalent neuropsychiatric condition in childhood. ADHD is a multifactorial trait with a strong genetic component. One neurodevelopmental hypothesis is that ADHD is associated with a lag in brain maturation. Sphingolipids are essential for brain development and neuronal functioning, but their role in ADHD pathogenesis is unexplored. We hypothesized that serum sphingolipid levels distinguish ADHD patients from unaffected subjects.

Methods: We characterized serum sphingolipid profiles of ADHD patients and two control groups: non-affected relatives and non-affected subjects without a family history of ADHD. Sphingolipids were measured by LC-MS/MS in 77 participants (28 ADHD patients, 28 related controls, and 21 unrelated controls). ADHD diagnosis was based on the Diagnostic and Statistical Manual of Mental Disorders (DSM IV-TR). Diagnostic criteria were assessed by two independent observers. Groups were compared by parametrical statistics.

Results: Serum sphingomyelins C16:0, C18:0, C18:1, C24:1, ceramide C24:0, and deoxy-ceramide C24:1 were significantly decreased in ADHD patients at $20-30 \%$ relative reductions. In our sample, decreased serum sphingomyelin levels distinguished ADHD patients with $79 \%$ sensitivity and $78 \%$ specificity.

Conclusions: Our results showed lower levels of all major serum sphingomyelins in ADHD. These findings may reflect brain maturation and affect neuro-functional pathways characteristic for ADHD.

Keywords: ADHD, sphingolipids, sphingomyelins, ceramides, biomarker, endophenotype

\section{Introduction}

Attention deficit-hyperactivity disorder (ADHD) is a neurobehavioral condition characterized by persistent, cross-situational and developmentally inappropriate levels of inattention, hyperactivity, and impulsiveness (American Psychiatric, 2000). ADHD affects 8-12\% of children of school age and is thus the most common pediatric neuropsychiatric disorder (Polanczyk et al., 2014).

Abbreviations: GLM, general linear model; ROC, receiver operating characteristic; SM, sphingomyelin. 
ADHD is considered a multifactorial trait in which genetic factors account for about $75-80 \%$ of the phenotypic variance and environmental factors, such as premature delivery, perinatal hypoxia, maternal smoking, alcohol consumption, and others, may contribute to the remaining 20-25\% (Thapar et al., 2013; Li et al., 2014; Polanczyk et al., 2014). The therapeutic effect of stimulants has led to wide acceptance of the dopaminergic hypothesis, a dysfunction in catecholaminergic/dopaminergic transmission, as a core mechanism for the disorder (Swanson et al., 2000; Tripp and Wickens, 2009), although associations identified between ADHD and monoaminergic gene variants account for a very modest risk effect (Li et al., 2014). A recent genome-wide association study suggested that exploration of alternative/complementary etiological factors related to neuritic outgrowth and maturation should be considered (Bralten et al., 2013). This is in line with neuroimaging studies, which increasingly suggest white/gray matter anomalies in prefrontal cortex, temporo-parietal regions, striatum, and cerebellum in ADHD patients (Casey et al., 2007; Silk et al., 2009; Helpern et al., 2011; Nagel et al., 2011; Peterson et al., 2011; de Zeeuw et al., 2012; Cortese et al., 2013; Greven et al., 2015). In this context, a prominent neurodevelopmental hypothesis proposes that ADHD involves a lag in brain maturation (Castellanos et al., 2002; Rubia, 2007; Shaw et al., 2007).

Sphingolipids, highly abundant in nervous tissue, affect neuronal and glial proliferation, differentiation, apoptosis as well as membrane permeability to $\mathrm{Ca}^{2+}$ and $\mathrm{K}^{+}$, relevant to the generation and propagation of the nervous impulse and neurotransmitter release (Colombaioni and Garcia-Gil, 2004; Gielen et al., 2006; Posse De Chaves and Sipione, 2010). Cell and animal models underscore the key function of sphingolipids in neurite growth and mass of cerebellum and forebrain (Hirabayashi and Furuya, 2008; Imgrund et al., 2009; Ginkel et al., 2012). Deficiency of ceramide synthase-2 that generates sphingolipids with $\mathrm{C} 22-\mathrm{C} 24$ fatty acyl chains, results in $50 \%$ loss of compacted myelin and $80 \%$ loss of CNS myelin basic protein (Imgrund et al., 2009). Similarly, a 60\% reduction of myelin-associated glycoprotein in cerebellum and forebrain characterizes mice deficient in ceramide synthase1, the enzyme that generates C18:0 sphingolipids. Hyperactive behavior characterizes mice deficient of ceramide synthase -6 that generates C16:0 sphingolipids (Ebel et al., 2013). In premature infants, supplementation of milk with sphingomyelins has been associated with significant improvements in attention and memory measurements at 18 months, suggesting positive effects on neurobehavioral development (Tanaka et al., 2013). Based on these observations and reported correlations between serum and CSF sphingolipids (Mielke et al., 2010, 2011) we hypothesized that serum sphingolipid levels distinguish ADHD patients from unaffected subjects. The exploration of sphingolipids as potential pathogenic factors and/or biomarkers for ADHD is new.

\section{Methods}

\section{Participants}

This case-control study was designed to compare serum sphingolipid profiles of ADHD patients with two independent control groups: unaffected first degree relatives of ADHD patients, sharing housing, and dietary patterns with the index cases and unaffected subjects with no family history of ADHD. We studied 77 subjects belonging to one of the following 3 groups: (1) ADHD patients ( $n=28$; 14 males), (2) unaffected first-degree relatives of ADHD patients [mother, father, or siblings of the index cases included in group (1), $n=28$; 4 males], and (3) unaffected participants without a family history of ADHD (also referred to as unaffected unrelated subjects or non-related controls, $n=21 ; 8$ males). Table 1 summarizes relevant characteristics of the three groups. ADHD diagnosis was based on the criteria of the Diagnostic and Statistical Manual of Mental Disorders in all cases (DSM IVTR) (American Psychiatric, 2000). Criteria were assessed by at least two independent observers (most commonly: parents and teachers), according to current guidelines (Seixas et al., 2012; Hauk, 2013). All participants were recruited from an ongoing study exploring the role of polyunsaturated fatty acids in ADHD pathogenesis (Henríquez-Henríquez et al., 2012). Participants with clinical evidence/anamnestic antecedent of major systemic and neurological illness and taking $\omega-3 / \omega-6$ supplementation were excluded from the study.

ADHD patients were between 5 and 18 years old (median = 12.7 years; 25 th percentile $=11$ years; 75 th percentile $=$ 17 years) and referred from general psychiatric/neurological and family medicine outpatient clinics serving a medium income area of urban Santiago, Chile. Seventeen patients were clinically classified as ADHD combined subtype, 10 patients as predominantly inattentive, and one patient as predominantly hyperactive-impulsive (American Psychiatric, 2000). This distribution agrees with the subtype prevalence generally described for clinically referred ADHD patients across the globe (Willcutt, 2012). All patients were treated with either d-amphetamine or methylphenidate in doses ranging $10-30 \mathrm{mg} /$ day. A medication free washout period of $24 \mathrm{~h}$ was required prior to blood analyses ( $\mathrm{t} 1 / 2$ for methylphenidate at 10-20 mg/kg: 2-4 h; t1/2 for d-amphetamine 6-12 h).

ADHD diagnosis was excluded from the unaffected groups (groups 2 and 3) by means of a brief clinical interview exploring DSM-IV-TR criteria (American Psychiatric, 2000). We required that unaffected relatives participating in this study were living and eating in the same home with the ADHD index case, to control dietary and other unknown environmental factors. Group 3 (consisting of unaffected participants without a family history of ADHD) were children recruited from a mediumincome school in the same urban area and healthy adults recruited from the same family medicine services at the time of general annual checkup. Family history of ADHD was anamnestically explored in group 3 considering first and second degree relatives. Ages of unaffected relatives (group 2) ranged from 10 to 64 years (median $=34$ years; 25th percentile $=$ 23.6 years; 75 th percentile $=43$ years). In group 3 (non-related controls) ages ranged from 7 to 51 years (median $=23.5$ years; 25th percentile $=12.3$ years; 75 th percentile $=35.6$ years) Because age and gender distributions significantly differ among the 3 groups ( $p=0.01$ and $p=0.001$, respectively), we controlled for those in all subsequent statistical analyses. 
TABLE 1 | Relevant clinical and epidemiological characteristics in the three groups studied.

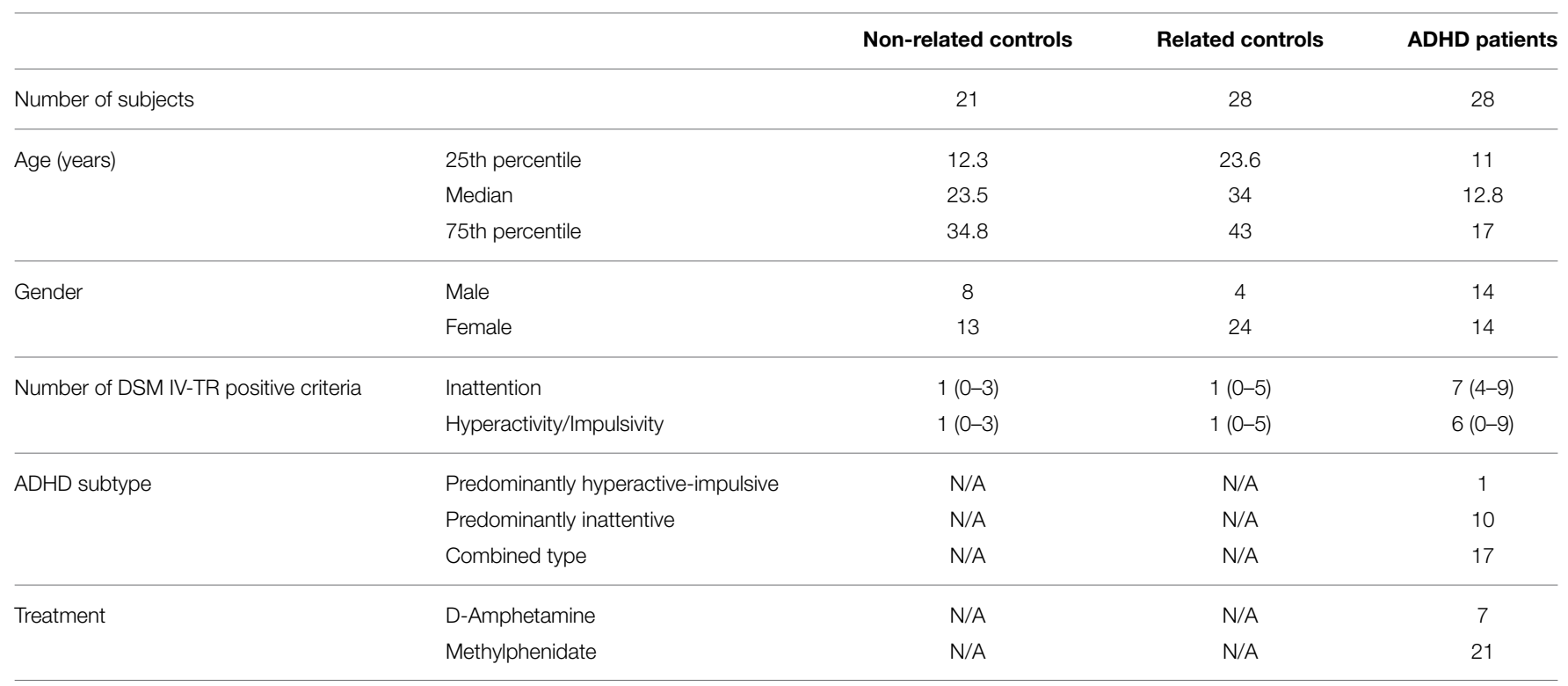

Age is described in terms of the percentiles 25th, 50th, and 75th due to non-Gaussian distribution of this variable inside the groups.

All procedures performed as part of this study have been approved by the Ethics Committee of Pontificia Universidad Católica de Chile. The study was fully explained to children and their parents, and they both agreed to participate by signing written consent and assent forms.

\section{Serum Sphingolipid Analysis}

Blood samples were obtained after an $8 \mathrm{~h}$ fast. Samples were centrifuged and obtained serum was stored at $-20^{\circ} \mathrm{C}$ until analysis.

Sphingolipid profiles include the following molecular species: (1) sphinganine-1-phosphate and sphingosine-1-phosphate; (2) ceramides C16:0, C18:0, C20:0, C22:0, C24:0, and C24:1; dihydroceramides C18, C18:1, C24:0, and C24:1; deoxyceramide C16:0 and C24:1 and deoxy-dihydroceramides C16:0 and C24:1; (3) sphingomyelins (SM) SM C16:0, SM C18:0, SM C18:1, and SM C24:1. Their relative location in the sphingolipid metabolic pathway is summarized in Figure 1. Sphingolipid levels were quantified by high performance liquid chromatography-triple quadrupole-tandem mass spectrometry (HPLC-MS/MS) on an Agilent 1200 HPLC system, equipped with an Agilent C18 column as reported (Bui et al., 2012). Briefly, sphingolipids were extracted overnight in a 1:30 v/v solution of diethylamide $10 \%$ /dichloromethanol: methanol 1:1, at room temperature using sphingomyelin $\mathrm{C} 12(0.120 \mu \mathrm{M})$ as internal standard. HPLC conditions were: Mobile phase A was methanol/water/chloroform/formic acid (55:40:5:0.4 v/v); Mobile phase B was methanol/acetonitrile/chloroform/formic acid (48:48:4:0.4 v/v). The chromatography column was first pre-equilibrated for $6 \mathrm{~s}$. After this period, the gradient gradually increased to $60 \%$ mobile phase B and $100 \%$ mobile phase B. $100 \%$ mobile phase $B$ was held for $1.9 \mathrm{~min}$. Flow rate was $0.6 \mathrm{ml} / \mathrm{min}$. Injection into the HPLC-MS/MS system was set at $3 \mu \mathrm{l}$. All measurements were performed in triplicates.

\section{Data Analysis}

The final serum level for each patient and analyte was calculated by averaging triplicate measurements. Subsequent statistical analyses were performed on these final levels. Average inter-assay imprecision for sphingolipid quantification was $8.7 \%$ (ranging from $2.5 \%$ for sphingomyelins to $12.3 \%$ for deoxyceramides).

\section{Statistical Analysis}

The normal distribution of individual sphingolipids was evaluated by the Kolmogorov-Smirnov test after outlier detection/rejection by the Tukey method (Tukey, 1977). All sphingolipids were normally distributed. Group differences were examined by general linear models (GLM) using sphingolipid levels as the dependent variable and "clinical group," "age," and "gender" as covariates. "Clinical group" was defined as a categorical variable with 3 levels: ADHD patients, unaffected relatives of $\mathrm{ADHD}$ patients and unaffected unrelated subjects. Post-hoc pair-wise comparisons were performed when significant effect for the variable "clinical group" was detected and significance was maintained as $p=0.05$. This approach was verified using the adaptive procedure described by Benjamini and Hochberg (BH-A) for controlling the False Discovery Rate (FDR) (Kromrey and Hogarty, 2002), with the same results in terms of the rejection of the null hypothesis. All analyses were conducted using SAS 9.2 software (SAS Institute, Cary, North Carolina).

To evaluate the ability of the assayed sphingolipids for correctly classifying ADHD affected and non-affected subjects, we performed Receiver Operating Characteristic (ROC) analyses (Fawcett, 2004), using the method of Delong et al for the calculation of the standard error of the area under the curve (DeLong et al., 1988). 

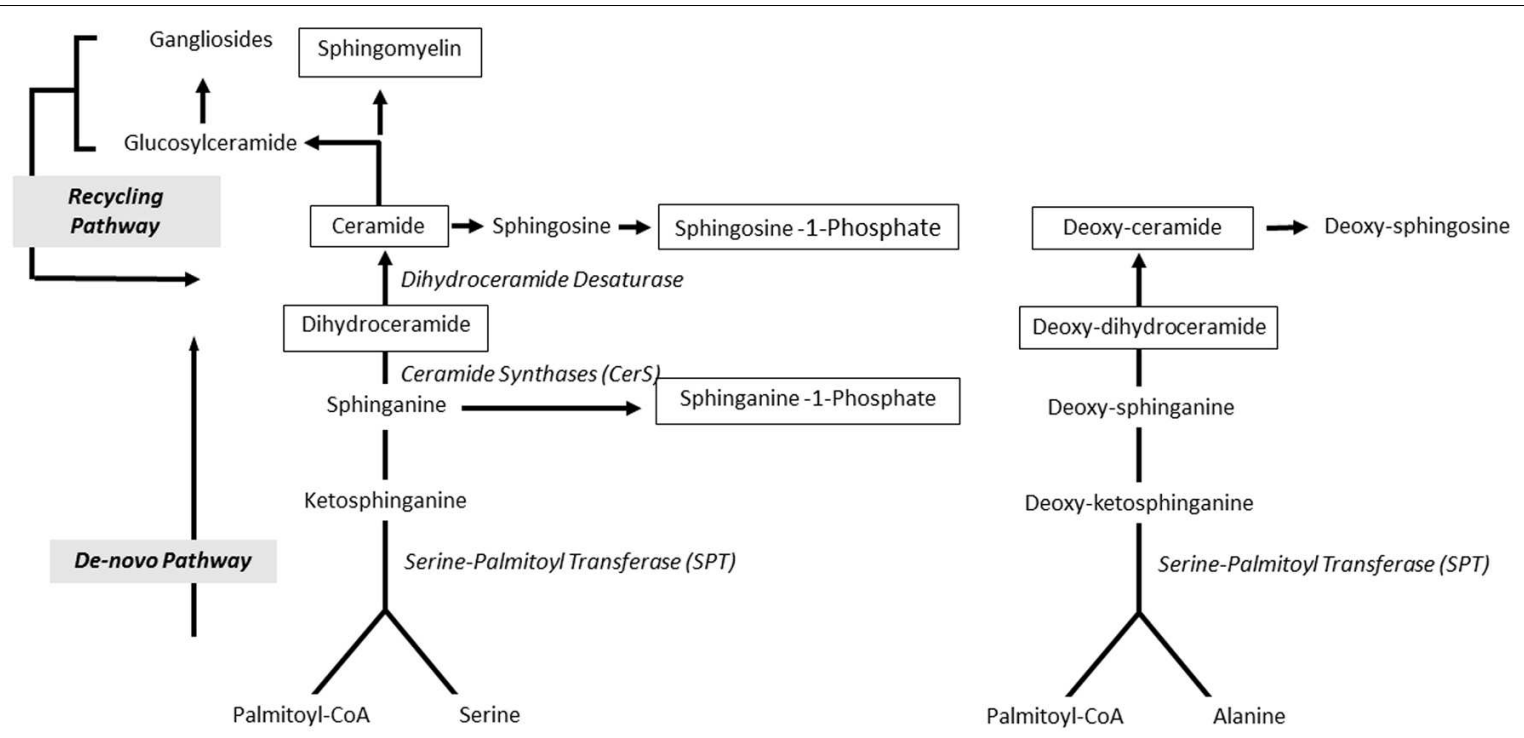

FIGURE 1 | Sphingolipid metabolic pathways. Ceramide is central in sphingolipid metabolism, produced by de novo and recycling pathways. Serine and palmitoyl-CoA are substrates of serine palmitoyl-transferase (SPT), the rate limiting enzyme of de novo synthesis that generates ketosphinganine from serine and palmitoyl-CoA (bottom). Ketosphinganine is reduced to form sphinganine that is $\mathrm{N}$-acylated by ceramide synthases with fatty acids of different chain lengths to form dihydroceramides.

Dihydroceramides are desaturated by dihydroceramide desaturase (DES) to generate ceramides. Ceramides can be metabolized to sphingomyelin or glycosphingolipids. SPT generates deoxyceramides when utilizing alanine instead of serine. Notably, deoxyceramides only originate from de novo synthesis. The recycling pathway generates ceramides from sphingomyelin and other complex sphingolipids. Ceramidases degrade ceramides and release sphingoid bases, which are reutilized for complex sphingolipid biosynthesis or phosphorylated to sphingosine-1-phosphate. Boxed compounds indicate molecular species assayed in this study.

\section{Results}

\section{Analysis of Sphingomyelins}

ADHD patients had lower serum levels of all assayed SM species. The "clinical group" effect was significant in all species after controlling by age and gender (SM C16:0 $p<0.0001$; $\mathrm{SM}$ C18:1 $p=0.05$; SM C18:0 $p=0.01$; SM 24:1 $p=$ 0.001 ) (Figure 2). Pairwise comparisons confirmed significant differences between ADHD patients and both control groups for all the species, except for sphingomyelin C18:1, where ADHD patients were significantly different only when compared to related controls. The biggest relative difference was observed for sphingomyelin C24:1, which was 21\% lower in ADHD patients. Relative differences in other species ranged from 13\% (SM C16:0) to $18 \%$ (SM C18:0).

\section{Analysis of Ceramides, Dihydroceramides, and Deoxy-ceramides}

Analysis of ceramides (C16:0, C18:0, C22:0, C24:1, and C24:0) showed 20\% lower ceramide C24:0 in ADHD patients compared to unaffected relatives (post-hoc pair-wise comparison $p=0.02$ ) (Figure 3). This difference was maintained when comparing ADHD patients with non-related controls (post-hoc pairwise comparison $p=0.04$ ). We explored dihydroceramide levels to evaluate if decreased ceramide C24:0 could originate from decreased de novo synthesis and found no differences between groups (data not shown). To further evaluate the

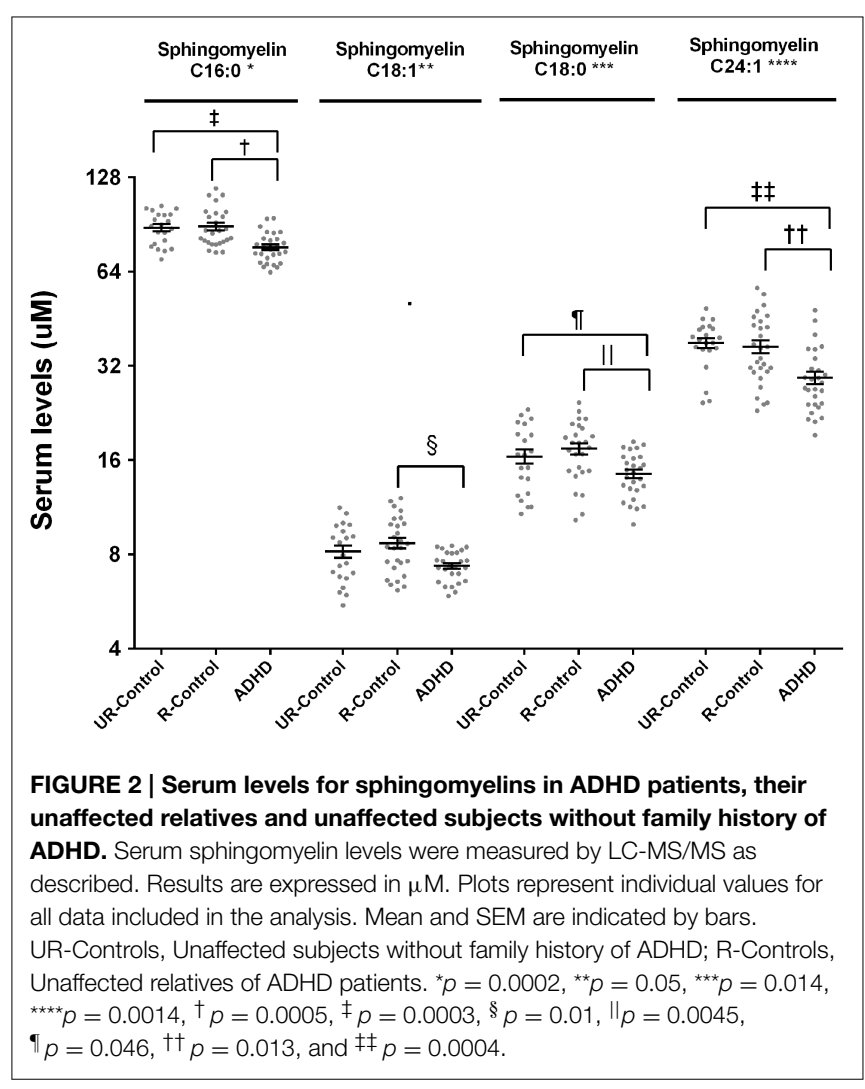




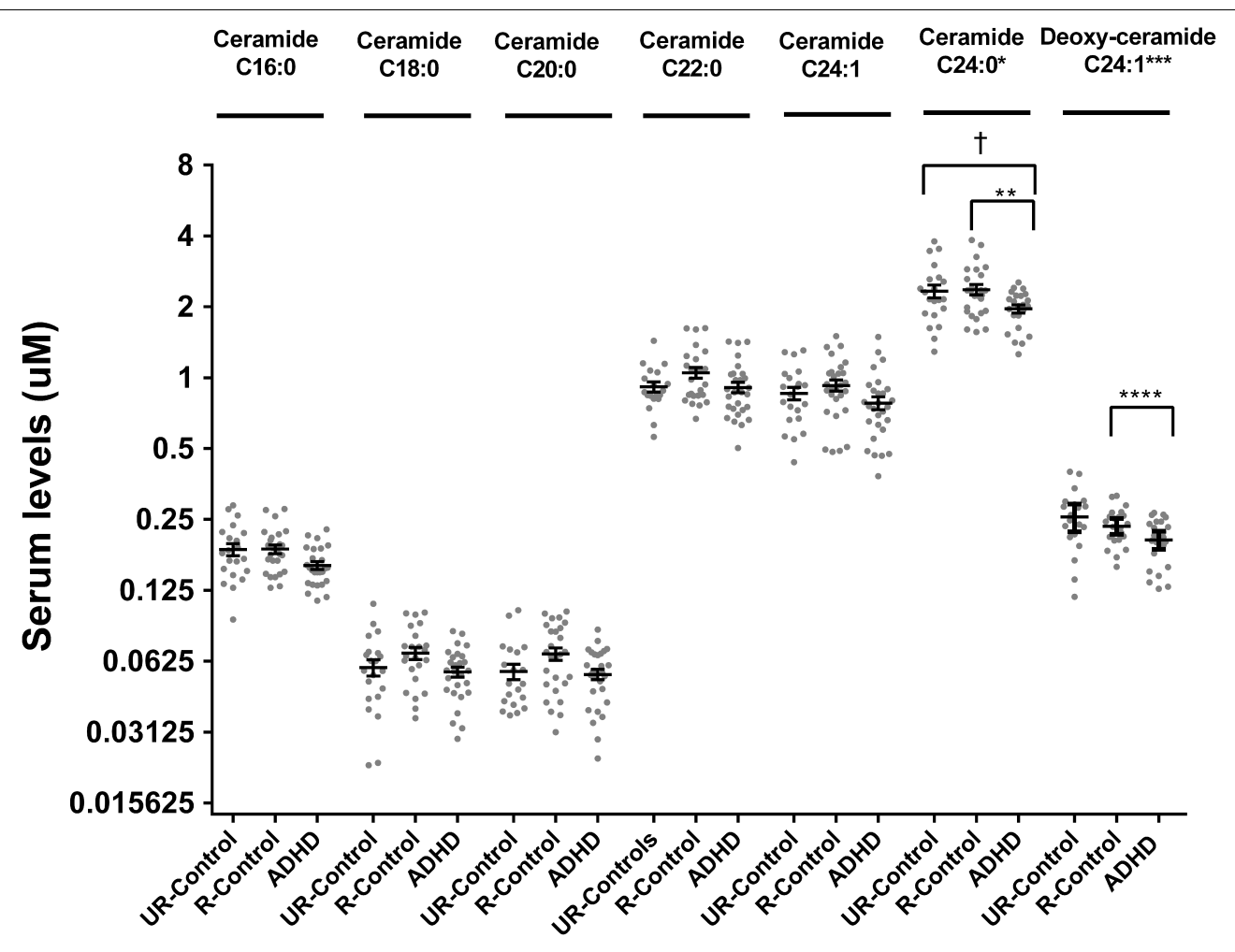

FIGURE 3 | Serum levels for ceramides and deoxy-ceramides in ADHD patients, their unaffected relatives and unaffected subjects without family history of ADHD. Serum ceramide levels were measured by LC-MS/MS as described. Results are expressed in $\mu \mathrm{M}$. Plots represent individual values for all data included in the analysis. Mean and SEM are indicated by bars. UR-Controls, Unaffected subjects without family history of ADHD; R-Controls, Unaffected relatives of ADHD patients. ${ }^{*} p=0.03$, ${ }^{* *} p=0.018,{ }^{* * *} p=0.003,{ }^{* * * *} p=0.01$, and ${ }^{\dagger} p=0.0411$. de-novo pathway we also measured deoxy-ceramides, which originate obligatory through the de-novo pathway (Figure 1). We measured the four most abundant species for which external standards are available (deoxy-dihydroceramides C16:0, C24:1, and deoxy-ceramides $\mathrm{C} 16: 0, \mathrm{C} 24: 1)$ and found deoxy-ceramide C24:1 to be $30 \%$ lower in ADHD patients compared to related controls (pairwise comparison $p=0.003$; $p$-value "clinical group" effect in GLM = 0.01) (Figure 3). A similar, but not significant trend was observed when comparing deoxy-ceramide C24:1 in ADHD patients to levels found in non-related controls (relative difference $=14 \%$; post-hoc pairwise comparison $p=0.1$ ).

\section{Sphingosine-1-Phosphate and Sphinganine-1-Phosphate Levels}

We evaluated levels of sphingosine-1-phosphate and sphinganine-1-phosphate among the three groups, of potential interest as sphingosine-1-phosphate has been linked to regulation of neuronal excitability and neurotransmitter release (Colombaioni and Garcia-Gil, 2004). Mean serum sphingosine1-phosphate was $1.55 \mu \mathrm{M}(\mathrm{SD}=0.38 \mu \mathrm{M})$ in ADHD patients. Concentrations did not differ $(p=0.48)$ from those found in unaffected relatives (mean $\pm \mathrm{SD}=1.44 \pm 0.38 \mu \mathrm{M}$ ) or unaffected controls without a family history of ADHD (mean \pm $\mathrm{SD}=1.59 \pm 0.38 \mu \mathrm{M})$. Mean serum sphinganine-1-phosphate was $0.23 \mu \mathrm{M}(\mathrm{SD}=0.008 \mu \mathrm{M})$ in $\mathrm{ADHD}$ patients, $0.2 \mu \mathrm{M}$ $(\mathrm{SD}=0.01 \mu \mathrm{M})$ in unaffected relatives and $0.22 \mu \mathrm{M}(\mathrm{SD}=$ $0.007 \mu \mathrm{M})$ in non-related controls. Sphinganine-1-phosphate concentrations were not different between groups $(p=0.27)$. These findings suggest that serum sphingoid bases are not different in ADHD patients.

\section{Low Serum Sphingolipids Distinguish ADHD Patients from Unaffected Subjects}

We built Receiver Operating Characteristic (ROC) curves to evaluate whether any of the assayed sphingolipids would discriminate between ADHD-affected and unaffected subjects with adequate levels of sensitivity and specificity. The best detection accuracy was obtained for total SM levels (the sum of all detected SM), with an AUC of 0.81 (CI: 0.71 to 0.89; $p<0.0001$ ), $79 \%$ sensitivity and $78 \%$ specificity (Figure 4). The estimated negative predictive value, considering 10\% ADHD prevalence, was $97 \%$.

\section{Sphingolipids as Potential Endophenotypes for ADHD}

To explore whether any of our findings can be considered as a candidate endophenotype (or intermediate phenotype) for $\mathrm{ADHD}$, we evaluated if they would fit an endophenotypecharacteristic stair-like distribution, with the highest frequency 


\section{A}
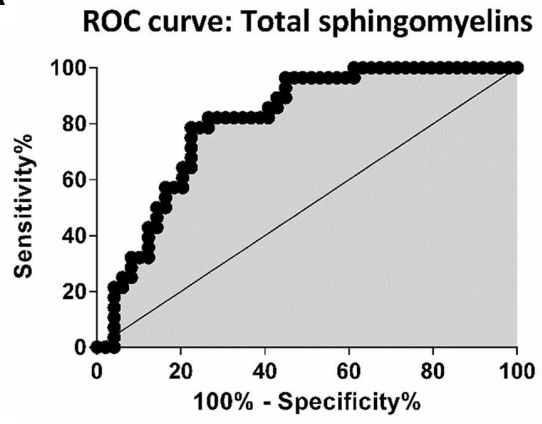

B

\begin{tabular}{c|c|c|}
\multicolumn{1}{c|}{} & \multicolumn{1}{c|}{ ADHD + } & ADHD - \\
\cline { 2 - 3 } $\begin{array}{c}\text { Above Cutoff } \\
\text { (Test }+)\end{array}$ & $\begin{array}{c}22 \\
\text { True Positives }\end{array}$ & $\begin{array}{c}11 \\
\text { False Positives }\end{array}$ \\
\cline { 2 - 3 } $\begin{array}{c}\text { Below Cutoff } \\
\text { (Test -) }\end{array}$ & False Negatives & True Negatives \\
\hline & & \\
\hline
\end{tabular}

FIGURE 4 | Receiver operating characteristic (ROC) curve and confusion matrix for total sphingomyelins. (A) Receiver operating characteristic curve $(\mathrm{ROC})$. The point of best discrimination was determined to be 134 uM total sphingomyelins. Total sphingomyelins refers to all assayed sphingomyelins. (B) Contingency table (confusion matrix) for total sphingomyelins. Total sphingomyelins $<134 \mu \mathrm{M}$ provide a sensitivity of $79 \%$ and specificity of $78 \%$. Considering a prevalence of $10 \%$ for ADHD, the negative predictive value for total sphingomyelins is $97 \%$.

of the marker among affected subjects, intermediate frequencies among unaffected subjects with positive family antecedents and the lowest frequencies among unaffected subjects without family antecedents of the condition. Based on the ROC curves, we chose the cut-off that best discriminated between ADHD and unaffected subjects for each analyte and built contingency tables that enabled us to evaluate the frequency of subjects below and above the cut-off inside the three clinical groups. The expected stair-like distribution was observed for SM C24:1 and deoxy-ceramide C24:1 ( $p$-values: $p<0.0001$, and $p=0.01$, respectively) (Figure 5). As a confirmatory approach and in order to adjust significances by age and gender effects, we also adjusted a GLM using the variable "clinical group" as a quantitative variable with three levels, being the highest for ADHD patients and the lowest for non-related controls, with similar results ( $p$-values: $p<0.001$ for SM C24:1 and $p=0.03$ for deoxy-ceramide C24:1).

\section{Discussion}

In this pilot study, we characterized sphingolipid serum profiles in ADHD patients and two control groups: (1) unaffected first degree relatives of ADHD patients who live and eat with their index ADHD case and (2) unaffected subjects without a family
A

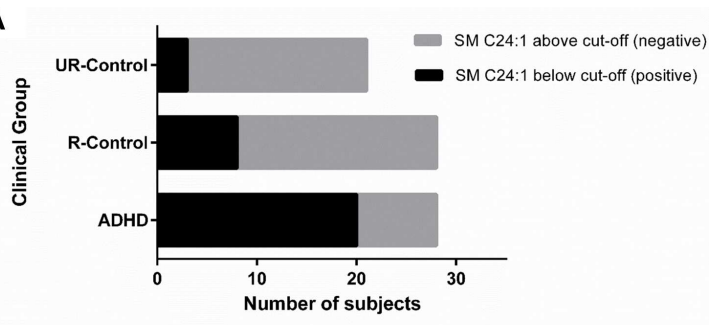

B

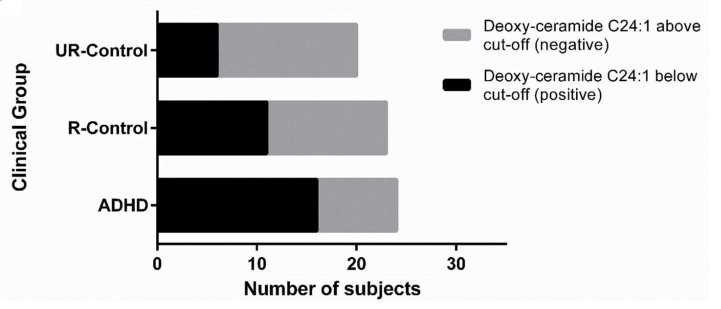

FIGURE 5 | SM C24:1 and deoxy-ceramide C24:1 may be ADHD endophenotypes. Contingency graphs showing the number of subjects below and above cutoff among ADHD patients, their unaffected relatives and non/related unaffected controls for (A) SM C24:1 and (B) deoxy-ceramide C24:1. UR-controls, Unaffected subjects without family history of ADHD; R-Controls, Unaffected relatives of ADHD patients.

history of ADHD among their first and second degree relatives. Sphingolipids and their metabolites are important for neuronal functioning (Buccoliero and Futerman, 2003; Colombaioni and Garcia-Gil, 2004), but serum concentrations have not been reported in the context of ADHD.

The major finding of our study is that serum levels for all major sphingomyelins and select ceramides are lower in ADHD patients compared to related -as well as to- unrelated controls. The biggest relative difference in sphingomyelin levels among the groups was observed for the long chain sphingomyelin C24:1, with a $21 \%$ reduction in the ADHD group when compared to the control groups. It is noteworthy that although more than 100 different subspecies of sphingomyelins have been described in human serum, the four species included in this study account for as much as $60 \%$ of total SM, while each one of the remaining non-assayed species account for about $0.5 \%$ of total SM (Quehenberger et al., 2010).

Figure 1 shows a schematic representation of sphingolipid metabolic pathways with emphasis on those metabolites that are relevant to our results. Ceramide can originate from de novo synthesis from serine and palmitoyl-CoA, or from the recycling pathway through catabolism of complex sphingolipids. Serinepalmitoyl transferase can alternatively utilize alanine instead of serine resulting in the synthesis of deoxyceramides. Degradation of ceramide yields sphingoid bases that can be phosphorylated to sphingosine-1-phosphate. Based on this scheme, potential causes for observed decreased serum sphingomyelins are (1) decreased sphingomyelin uptake or synthesis or (2) increased sphingomyelinase activity. Neither of these mechanisms was specifically evaluated in the current study.

Ceramide C24:0 was decreased 20\% and deoxy-ceramide C24:1 -which originates exclusively from the de-novo pathway- 
was decreased $30 \%$ in $\mathrm{ADHD}$ patients. Notably, the low levels of ceramide C24:0 and deoxy-ceramide C24:1 in ADHD patients are not paralleled by a decrease in any dihydroceramide, suggesting that ceramide synthase- 2 activity (which generates dihydroceramides with acyl chains C22-24) might not be impaired in ADHD. Comparable concentrations of all dihydroceramide species also suggest that low ceramide $\mathrm{C} 24: 0$ and deoxy-ceramide C24:1 are not compensated by increased synthesis of other species through the de-novo pathway (see Figure 1). Thus, potential causes for observed decreased ceramides are (1) selectively decreased activity of dihydroceramide desaturase (DES) that converts dihydroceramides to ceramides, or (2) increased conversion of ceramide C24:0 into sphingosine, ceramide-1phosphate or glucosylceramides, which were not evaluated. Decreased ceramide C24:0 might also be caused by decreased sphingomyelin C24:0, not measured in this study. Such a mechanism, however, cannot explain decreased deoxy-ceramide C24:1, since this ceramide is not a degradation product of sphingomyelin or any other more complex sphingolipids and must be generated de-novo. Notably, decreased nervonic acid (C24:1) has been reported in red blood cells of ADHD patients (Chen et al., 2004).

Whether serum sphingolipid profiles reflect brain sphingolipid metabolism is not known. For patients with HIV and Alzheimer's disease, a significant correlation between ceramide and sphingomyelin levels in plasma and CSF have been reported (Mielke et al., 2010, 2011), but no studies have explored these correlations in the general population. Future research is necessary to evaluate if plasma sphingolipid levels can be related to changes in CSF and brain tissue in ADHD.

The interpretation and extrapolation of our results must consider some limitations. First, cases and controls are not agegender matched and we observe significant differences in the distributions for these variables among the groups (see Table 1). As a consequence, we are not able to completely rule-out a confounding effect for age and gender, even though we controlled by these variables in all subsequent statistical analyses. To date, there is very limited data about physiological changes associated to age and gender in serum sphingolipids. A recent study describes a specific gender effect for the serum levels of several sphingomyelins, with higher values for women (Ishikawa et al., 2014), but we did not observe significant gender effects in anyone of the studied ceramide/sphingomyelin species in our sample (Table 2). The same study did not show age specific effects for any of the sphingolipids that we report to be significantly associated with ADHD. We explored eventual correlations between age and serum sphingolipid levels in our sample and did not find significant correlations for anyone of the analytes included in this study, except for ceramide C24:1, which did not present significant differences between ADHD patients and controls (Table 3). We also performed a subsample analysis considering all participants from 5 to 18 years old (mean age ADHD group $=$ 12.3 years $95 \% \mathrm{CI}=11.25-13.42$; mean age controls $=12.2$ years $95 \% \mathrm{CI}=10.87-13.7 p=0.72$ ) and were able to confirm our observations in terms of significantly lower levels for all sphingomyelins (SM C16:0 $p=0.0028$; SM C18:0 $p=0.0088$;
TABLE 2 | Gender effects for the studied ceramides and sphingomyelins.

\begin{tabular}{lcc}
\hline Analyte & $\boldsymbol{F}$-value & $\boldsymbol{p}$-value \\
\hline SM C16:0 & 0.09 & 0.77 \\
SM C18:0 & 0.04 & 0.85 \\
SM C18:1 & 1.89 & 0.18 \\
SM C24:1 & 0.12 & 0.73 \\
C16:0 & 0.01 & 0.93 \\
C18:0 & 1.39 & 0.24 \\
C20:0 & 2.14 & 0.13 \\
C22:0 & 0.35 & 0.55 \\
C24:0 & 0.73 & 0.39 \\
C24:1 & 0,19 & 0.67 \\
Deoxy-ceramide C24:1 & 0.12 & 0.73
\end{tabular}

Reported effects are controlled by age and clinical group.

SM C18:1 $p=0.018$; SM C24:1 $p=0.0038)$. Of note, we also performed ROC analysis for total SM levels using an age-matched subsample, finding sensitivity and specificity values comparable with those reported for the complete sample $\left(\mathrm{AUC}_{\text {subsample }}=\right.$ 0.82 CI: $0.66-0.93, p<0.0001 ; 82 \%$ sensitivity and $78 \%$ specificity), suggesting that results are not affected by age. We also observed the same i.e., decreased albeit non-significant trends for ceramide C24:0 and deoxy-ceramide C24:1 in the sub-sample analyses (C24:1 $F=1.1 p=0.3$ and deoxy-ceramide $\mathrm{C} 24: 1 F=$ $2.36 p=0.14$, respectively). As a second limitation, none of our patients were treatment naïve and we cannot rule-out that our findings correspond to a chronic treatment effect instead of being an actual pathogenic marker/factor. Acute treatment effects, on the other hand, were prevented by requiring a $24 \mathrm{~h}$ washout period for medication. Finally, our restricted sample size did not allow us to perform analyses by clinical subtype and we are not able to distinguish whether the reported results are applicable to all clinical subgroups or were driven by a specific clinical subtype. We also might be underpowered for detecting some differences of eventual clinical importance. As an example, taking into account the overall variation of our data, we estimate that our study is not able to detect differences less than $20 \%$ for the majority of the ceramides. In light of the potential relevance of the results reported by this pilot study, future research addressing these limitations and including the exploration of additional confounders, such as co-morbidities, is mandatory.

Strengths of our study include (1) the presence of a comparison group of first degree relatives that live and eat with the index case to control for a potential "diet" effect and for other unknown environmental factors and (2) the inclusion of an additional control group of unaffected subjects without a family history of ADHD to assess replication of findings. This is relevant because differences observed between ADHD patients and their relatives could reveal either a true characteristic of ADHD patients, or reflect compensatory mechanisms exerted by unaffected relatives to constitutional risk factors shared with ADHD patients.

In addition, the experimental design of this study allows to explore whether sphingolipid levels fulfill the distribution criteria proposed by Gottesman and Gould for endophenotypes 
TABLE 3 | Age-serum level correlations for the studied ceramides and sphingomyelins.

\begin{tabular}{lcc}
\hline Analyte & Pearson correlation coefficient & $\boldsymbol{p}$-value \\
\hline SM C16:0 & 0.14 & 0.91 \\
SM C18:0 & -0.02 & 0.88 \\
SM C18:1 & 0.07 & 0.57 \\
SM C24:1 & 0.15 & 0.2 \\
C16:0 & 0.17 & 0.14 \\
C18:0 & 0.07 & 0.54 \\
C20:0 & 0.14 & 0.25 \\
C22:0 & 0.22 & 0.07 \\
C24:0 & 0.11 & 0.38 \\
C24:1 & 0.25 & 0.03 \\
Deoxy-ceramide C24:1 & 0.016 & 0.89 \\
\hline
\end{tabular}

Reported correlations correspond to partial correlations using gender and presence/absence of $A D H D$ as co-variants.

of psychiatric disorders (Gottesman and Gould, 2003). Endophenotypes are quantifiable markers of genetic liability for non-mendelian complex diseases, which constitute a more "direct" expression of the gene effect than the disorder itself, since they are influenced by fewer genetic and environmental variables. They do not need to be specific, since they might signal common pathogenic mechanisms between related disorders. Our results suggest that sphingomyelin C24:1 and deoxy-ceramide C24:1 qualifies for the expected endophenotype distribution and should be further explored as potential endophenotypes

\section{References}

American Psychiatric, A. (2000). Diagnostic and Statistical Manual of Mental Disorders, 4th Edn., Text Revision (DSM-IV-TR). Washington, DC: American Psychiatric Publishing.

Asherson, P., Zhou, K., Anney, R. J., Franke, B., Buitelaar, J., Ebstein, R., et al. (2008). A high-density SNP linkage scan with 142 combined subtype ADHD sib pairs identifies linkage regions on chromosomes 9 and 16. Mol. Psychiatry 13, 514-521. doi: 10.1038/sj.mp.4002140

Bralten, J., Franke, B., Waldman, I., Rommelse, N., Hartman, C., Asherson, P., et al. (2013). Candidate genetic pathways for attention-deficit/hyperactivity disorder (ADHD) show association to hyperactive/impulsive symptoms in children with ADHD. J. Am. Acad. Child Adolesc. Psychiatry 52, 1204-1212.e1. doi: 10.1016/j.jaac.2013.08.020

Buccoliero, R., and Futerman, A. H. (2003). The roles of ceramide and complex sphingolipids in neuronal cell function. Pharmacol. Res. 47, 409-419. doi: 10.1016/S1043-6618(03)00049-5

Bui, H. H., Leohr, J. K., and Kuo, M. S. (2012). Analysis of sphingolipids in extracted human plasma using liquid chromatography electrospray ionization tandem mass spectrometry. Anal. Biochem. 423, 187-194. doi: 10.1016/j.ab.2012.01.027

Casey, B. J., Epstein, J. N., Buhle, J., Liston, C., Davidson, M. C., Tonev, S. T., et al. (2007). Frontostriatal connectivity and its role in cognitive control in parent-child dyads with ADHD. Am. J. Psychiatry 164, 1729-1736. doi: 10.1176/appi.ajp.2007.06101754

Castellanos, F. X., Lee, P. P., Sharp, W., Jeffries, N. O., Greenstein, D. K., Clasen, L. S., et al. (2002). Developmental trajectories of brain volume abnormalities in children and adolescents with attention-deficit/hyperactivity disorder. JAMA 288, 1740-1748. doi: 10.1001/jama.288.14.1740 for ADHD. Notably, regions containing genes encoding key enzymes in the sphingolipid metabolism i.e., serine-palmitoyl transferase (SPTLC1, SPTLC3, SPTSSB) and sphingomyelinases (SMPD1, SMPD4, SMPD3A), have been mapped in association with ADHD (Asherson et al., 2008; Romanos et al., 2008; Rommelse et al., 2008).

In conclusion, our results show decreased serum levels of sphingomyelins SM C16:0, SM C18:0, SM C18:1, and SM C24:1, of ceramide C24:0 and deoxy-ceramide C24:1 in ADHD patients compared to related and unrelated controls. This is the first study of serum sphingolipids in ADHD patients. Our data suggest that sphingomyelin SM C24:1 and deoxy-ceramide C24:1 are potential markers for ADHD (endophenotypes) that could reflect pathomechanisms relevant to the disorder.

\section{Author Contributions}

Study/Experiments design: MH, SS, and TQ. Experiment execution: $\mathrm{MH}$ and BK. Data analysis/Statistical analysis: $\mathrm{MH}$ and BK. Interpretation of results and discussion: $\mathrm{MH}, \mathrm{TW}$, and RD. Contributed reagents/materials/analysis tools: $M H, T W, S S$, and TQ. Elaboration of the manuscript: $\mathrm{MH}$ and TW. Critic lecture of the manuscript: TW, RD, SS, and TQ.

\section{Acknowledgments}

This study was financed by funds from the Department of Pathology, Columbia University Global Grant (both for TW) and a stipend from the School of Medicine, Pontificia Universidad Católica de Chile, Chile (MH).

Chen, J. R., Hsu, S. F., Hsu, C. D., Hwang, L. H., and Yang, S. C. (2004). Dietary patterns and blood fatty acid composition in children with attentiondeficit hyperactivity disorder in Taiwan. J. Nutr. Biochem. 15, 467-472. doi: 10.1016/j.jnutbio.2004.01.008

Colombaioni, L., and Garcia-Gil, M. (2004). Sphingolipid metabolites in neural signalling and function. Brain Res. Brain Res. Rev. 46, 328-355. doi: 10.1016/j.brainresrev.2004.07.014

Cortese, S., Imperati, D., Zhou, J., Proal, E., Klein, R. G., Mannuzza, S., et al. (2013). White matter alterations at 33-year follow-up in adults with childhood attention-deficit/hyperactivity disorder. Biol. Psychiatry 74, 591-598. doi: 10.1016/j.biopsych.2013.02.025

DeLong, E. R., DeLong, D. M., and Clarke-Pearson, D. L. (1988). Comparing the areas under two or more correlated receiver operating characteristic curves: a nonparametric approach. Biometrics 44, 837-845. doi: 10.2307/2531595

de Zeeuw, P., Mandl, R. C., Hulshoff Pol, H. E., Van Engeland, H., and Durston, S. (2012). Decreased frontostriatal microstructural organization in attention deficit/hyperactivity disorder. Hum. Brain Mapp. 33, 1941-1951. doi: 10.1002/hbm.21335

Ebel, P., Vom Dorp, K., Petrasch-Parwez, E., Zlomuzica, A., Kinugawa, K., Mariani, J., et al. (2013). Inactivation of ceramide synthase 6 in mice results in an altered sphingolipid metabolism and behavioral abnormalities. J. Biol. Chem. 288, 21433-21447. doi: 10.1074/jbc.M113.479907

Fawcett, T. (2004). ROC graphs: notes and practical considerations for researchers. Mach. Learn. 31, 1-38.

Gielen, E., Baron, W., Vandeven, M., Steels, P., Hoekstra, D., and Ameloot, M. (2006). Rafts in oligodendrocytes: evidence and structure-function relationship. Glia 54, 499-512. doi: 10.1002/glia.20406

Ginkel, C., Hartmann, D., Vom Dorp, K., Zlomuzica, A., Farwanah, H., Eckhardt, M., et al. (2012). Ablation of neuronal ceramide synthase 1 
in mice decreases ganglioside levels and expression of myelin-associated glycoprotein in oligodendrocytes. J. Biol. Chem. 287, 41888-41902. doi: 10.1074/jbc.M112.413500

Gottesman, I. I., and Gould, T. D. (2003). The endophenotype concept in psychiatry: etymology and strategic intentions. Am. J. Psychiatry 160, 636-645. doi: 10.1176/appi.ajp.160.4.636

Greven, C. U., Bralten, J., Mennes, M., O’Dwyer, L., van Hulzen, K. J., Rommelse, N., et al. (2015). Developmentally stable whole-brain volume reductions and developmentally sensitive caudate and putamen volume alterations in those with attention-deficit/hyperactivity disorder and their unaffected siblings. JAMA Psychiatry 72, 490-499. doi: 10.1001/jamapsychiatry.2014.3162

Hauk, L. (2013). AAP releases guideline on diagnosis, evaluation, and treatment of ADHD. Am. Fam. Physician 87, 61-62.

Helpern, J. A., Adisetiyo, V., Falangola, M. F., Hu, C., Di Martino, A., Williams, K., et al. (2011). Preliminary evidence of altered gray and white matter microstructural development in the frontal lobe of adolescents with attentiondeficit hyperactivity disorder: a diffusional kurtosis imaging study. J. Magn. Reson. Imaging 33, 17-23. doi: 10.1002/jmri.22397

Henríquez-Henríquez, M., Solari, S., Vargas, G., Vásquez, L., Allende, F., Castañon, C., et al. (2012). Omega-3 long chain polyunsaturated fatty acids and fatty acid desaturase activity ratios as eventual endophenotypes for ADHD. J. Atten. Disord. doi: 10.1177/1087054712461175. [Epub ahead of print].

Hirabayashi, Y., and Furuya, S. (2008). Roles of 1-serine and sphingolipid synthesis in brain development and neuronal survival. Prog. Lipid Res. 47, 188-203. doi: 10.1016/j.plipres.2008.01.003

Imgrund, S., Hartmann, D., Farwanah, H., Eckhardt, M., Sandhoff, R., Degen, J., et al. (2009). Adult ceramide synthase 2 (CERS2)-deficient mice exhibit myelin sheath defects, cerebellar degeneration, and hepatocarcinomas. J. Biol. Chem. 284, 33549-33560. doi: 10.1074/jbc.M109.031971

Ishikawa, M., Maekawa, K., Saito, K., Senoo, Y., Urata, M., Murayama, M., et al. (2014). Plasma and serum lipidomics of healthy white adults shows characteristic profiles by subjects' gender and age. PLOS ONE 9:e91806. doi: 10.1371/journal.pone.0091806

Kromrey, J. D., and Hogarty, K. Y. (2002). FDR_TEST: A SAS Macro for Calculating New Methods of Error Control in Multiple Hypothesis Testing. Savannah, GA: Southeast SAS Users Group.

Li, Z., Chang, S. H., Zhang, L. Y., Gao, L., and Wang, J. (2014). Molecular genetic studies of ADHD and its candidate genes: a review. Psychiatry Res. 219, 10-24. doi: 10.1016/j.psychres.2014.05.005

Mielke, M. M., Bandaru, V. V., McArthur, J. C., Chu, M., and Haughey, N. J. (2010). Disturbance in cerebral spinal fluid sphingolipid content is associated with memory impairment in subjects infected with the human immunodeficiency virus. J. Neurovirol. 16, 445-456. doi: 10.3109/13550284.2010.525599

Mielke, M. M., Haughey, N. J., Bandaru, V. V., Weinberg, D. D., Darby, E., Zaidi, N., et al. (2011). Plasma sphingomyelins are associated with cognitive progression in Alzheimer's disease. J. Alzheimers Dis. 27, 259-269. doi: 10.3233/JAD-2011-110405

Nagel, B. J., Bathula, D., Herting, M., Schmitt, C., Kroenke, C. D., Fair, D., et al. (2011). Altered white matter microstructure in children with attentiondeficit/hyperactivity disorder. J. Am. Acad. Child Adolesc. Psychiatry 50, 283-292. doi: 10.1016/j.jaac.2010.12.003

Peterson, D. J., Ryan, M., Rimrodt, S. L., Cutting, L. E., Denckla, M. B., Kaufmann, W. E., et al. (2011). Increased regional fractional anisotropy in highly screened attention-deficit hyperactivity disorder (ADHD). J. Child Neurol. 26, 1296-1302. doi: 10.1177/0883073811405662

Polanczyk, G. V., Willcutt, E. G., Salum, G. A., Kieling, C., and Rohde, L. A. (2014). ADHD prevalence estimates across three decades: an updated systematic review and meta-regression analysis. Int. J. Epidemiol. 43, 434-442. doi: 10.1093/ije/dyt261
Posse de Chaves, E., and Sipione, S. (2010). Sphingolipids and gangliosides of the nervous system in membrane function and dysfunction. FEBS Lett. 584, 1748-1759. doi: 10.1016/j.febslet.2009.12.010

Quehenberger, O., Armando, A. M., Brown, A. H., Milne, S. B., Myers, D. S., Merrill, A. H., et al. (2010). Lipidomics reveals a remarkable diversity of lipids in human plasma. J. Lipid Res. 51, 3299-3305. doi: 10.1194/jlr.M 009449

Romanos, M., Freitag, C., Jacob, C., Craig, D. W., Dempfle, A., Nguyen, T. T., et al. (2008). Genome-wide linkage analysis of ADHD using high-density SNP arrays: novel loci at 5q13.1 and 14q12. Mol. Psychiatry 13, 522-530. doi: $10.1038 / \mathrm{mp} .2008 .12$

Rommelse, N. N., Arias-Vásquez, A., Altink, M. E., Buschgens, C. J., Fliers, E., Asherson, P., et al. (2008). Neuropsychological endophenotype approach to genome-wide linkage analysis identifies susceptibility loci for ADHD on 2q21.1 and 13q12.11. Am. J. Hum. Genet. 83, 99-105. doi: 10.1016/j.ajhg.2008. 06.006

Rubia, K. (2007). Neuro-anatomic evidence for the maturational delay hypothesis of ADHD. Proc. Natl. Acad. Sci. U.S.A. 104, 19663-19664. doi: 10.1073/pnas.0710329105

Seixas, M., Weiss, M., and Müller, U. (2012). Systematic review of national and international guidelines on attention-deficit hyperactivity disorder. J. Psychopharmacol. 26, 753-765. doi: 10.1177/0269881111412095

Shaw, P., Eckstrand, K., Sharp, W., Blumenthal, J., Lerch, J. P., Greenstein, D., et al. (2007). Attention-deficit/hyperactivity disorder is characterized by a delay in cortical maturation. Proc. Natl. Acad. Sci. U.S.A. 104, 19649-19654. doi: 10.1073/pnas.0707741104

Silk, T. J., Vance, A., Rinehart, N., Bradshaw, J. L., and Cunnington, R. (2009). White-matter abnormalities in attention deficit hyperactivity disorder: a diffusion tensor imaging study. Hum. Brain Mapp. 30, 2757-2765. doi: $10.1002 / \mathrm{hbm} .20703$

Swanson, J. M., Flodman, P., Kennedy, J., Spence, M. A., Moyzis, R., Schuck, S., et al. (2000). Dopamine genes and ADHD. Neurosci. Biobehav. Rev. 24, 21-25. doi: 10.1016/S0149-7634(99)00062-7

Tanaka, K., Hosozawa, M., Kudo, N., Yoshikawa, N., Hisata, K., Shoji, H., et al. (2013). The pilot study: sphingomyelin-fortified milk has a positive association with the neurobehavioural development of very low birth weight infants during infancy, randomized control trial. Brain Dev. 35, 45-52. doi: 10.1016/j.braindev.2012.03.004

Thapar, A., Cooper, M., Eyre, O., and Langley, K. (2013). What have we learnt about the causes of ADHD? J. Child Psychol. Psychiatry 54, 3-16. doi: 10.1111/j.1469-7610.2012.02611.x

Tripp, G., and Wickens, J. R. (2009). Neurobiology of ADHD. Neuropharmacology 57, 579-589. doi: 10.1016/j.neuropharm.2009.07.026

Tukey, J. (1977). Exploratory Data Analysis. Reading, MA: Addison-Wesley.

Willcutt, E. G. (2012). The prevalence of DSM-IV attention-deficit/hyperactivity disorder: a meta-analytic review. Neurotherapeutics 9, 490-499. doi: $10.1007 / \mathrm{s} 13311-012-0135-8$

Conflict of Interest Statement: The authors declare that the research was conducted in the absence of any commercial or financial relationships that could be construed as a potential conflict of interest.

Copyright (๑) 2015 Henríquez-Henríquez, Solari, Quiroga, Kim, Deckelbaum and Worgall. This is an open-access article distributed under the terms of the Creative Commons Attribution License (CC BY). The use, distribution or reproduction in other forums is permitted, provided the original author(s) or licensor are credited and that the original publication in this journal is cited, in accordance with accepted academic practice. No use, distribution or reproduction is permitted which does not comply with these terms. 